TITLE:

\title{
Fabrication and evaluation via nuclear quadrupole resonance of a palm cubic-anvil pressure cell
}

\section{$\operatorname{AUTHOR(S):~}$}

Nakagawa, Satoshi; Gochi, Jun; Kuwayama, Takanori; Nagasaki, Shoko; Takahashi, Teruo; Cheng, Jinguang; Uwatoko, Yoshiya; Fujiwara, Naoki

\section{CITATION:}

Nakagawa, Satoshi ... [et al]. Fabrication and evaluation via nuclear quadrupole resonance of a palm cubic-anvil pressure cell. Review of Scientific Instruments 2020, 91(7): 073907.

\section{ISSUE DATE:}

2020-07-01

URL:

http://hdl.handle.net/2433/252828

\section{RIGHT:}

This article may be downloaded for personal use only. Any other use requires prior permission of the author and AIP Publishing. This article appeared in 'Review of Scientific Instruments' 91, 073907 (2020) and may be found at

https://aip.scitation.org/doi/10.1063/5.0012015; The full-text file will be made open to the public on 1 July 2021 in accordance with publisher's 'Terms and Conditions for SelfArchiving' 


\title{
Fabrication and evaluation via nuclear quadrupole resonance of a palm cubic-anvil pressure cell
}

Cite as: Rev. Sci. Instrum. 91, 073907 (2020); doi: 10.1063/5.0012015

Submitted: 27 April 2020 - Accepted: 26 June 2020 •

Published Online: 14 July 2020

Satoshi Nakagawa, ${ }^{1}$ Jun Gochi, ${ }^{2}$ Takanori Kuwayama, Shoko Nagasaki, ${ }^{2}$ Teruo Takahashi, ${ }^{1}$ Jinguang Cheng, Yoshiya Uwatoko, ${ }^{2}$ (D) and Naoki Fujiwara ${ }^{1, a)}$ (D)

\begin{abstract}
AFFILIATIONS
${ }^{1}$ Graduate School of Human and Environmental Studies, Kyoto University, Nihonmatsu, Sakyo-Ku, Kyoto 606-8501, Japan

${ }^{2}$ ISSP University of Tokyo, 5-1-5 Kashiwanoha, Kashiwa 277-8581, Japan

${ }^{3}$ Beijing National Laboratory for Condensed Matter Physics and Institute of Physics, Chinese Academy of Sciences, Beijing 100190, China
\end{abstract}

\begin{abstract}
a) Author to whom correspondence should be addressed: fujiwara.naoki.7e@kyoto-u.ac.jp
\end{abstract}

\begin{abstract}
A "palm" cubic-anvil pressure cell (PCAC) having an outer diameter of $60 \mathrm{~mm}$, the smallest cubic-anvil cell to date, was fabricated to insert in a large-bore superconducting magnet. The pressure cell has a sample space of $\phi 2.5 \times 1.5 \mathrm{~mm}^{2}$, which is fairly large for a pressure cell that can reach a high pressure above $4 \mathrm{GPa}$. Pressure homogeneity was monitored from the ${ }^{63} \mathrm{Cu}$ nuclear-quadrupole-resonance linewidth of $\mathrm{Cu}_{2} \mathrm{O}$ up to $6.7 \mathrm{GPa}$. The linewidth first increased with increasing pressure up to $4 \mathrm{GPa}$ and then saturated above $4 \mathrm{GPa}$. The pressure homogeneity was better than that of a piston-cylinder pressure cell. The PCAC is advantageous because a large sample space and high pressure homogeneity are secured even at high pressures.
\end{abstract}

Published under license by AIP Publishing. https://doi.org/10.1063/5.0012015

\section{INTRODUCTION}

In the field of strongly correlated electron systems, one of the most intriguing topics is the quantum critical-phase transition. Unlike a conventional thermally induced phase transition, this phase transition is driven via quantum fluctuations at zero temperature, leading to an unconventional ground state even at finite temperatures. The quantum-phase transition is usually achieved by changing non-thermal tuning parameters, such as doping level and pressure. Compared with the former, the latter is advantageous as the former can induce local disturbance around the dopant. To date, not a few novel pressure-induced or enhanced phenomena, including superconductivity, have been discovered in $d$ - or $f$-electron systems. Among them, Fe-based compounds are interesting because of their high $T_{c}$ under pressure. ${ }^{1-5}$ However, they have been investigated mainly by using resistivity measurements. A small sample is sufficient for resistivity measurements, which allows one to measure at significantly high pressures. For other techniques requiring a large volume, there is always a trade-off between high pressure and large sample space. To date, a variety of pressure cells have been developed, depending on experimental techniques and target materials. ${ }^{6}$ For example, piston-cylinder, ${ }^{7}$ opposed-anvil, ${ }^{8-10}$ cubic-anvil, ${ }^{11}$ and diamond-anvil cells ${ }^{12-16}$ have been used for nuclear magnetic resonance (NMR) and nuclear quadrupole resonance (NQR). The first three types of cells were often used in studying the strongly correlated electron systems. However, the diamond-anvil cell has been available only for detecting strong ${ }^{1} \mathrm{H}$ or ${ }^{19} \mathrm{~F}$ signals because of its tiny sample space, although it can generate the highest pressure among them.

At low pressures, a piston-cylinder cell has been most widely used for various experiments because it contains a large sample space. Its typical size is $\phi 4 \times 10 \mathrm{~mm}^{2}$ or larger at the initial setup. A conventional hybrid tungsten-carbide (WC) piston-cylinder cell can generate $2.7 \mathrm{GPa}-3 \mathrm{GPa}$. To date, pressures have reached $4 \mathrm{GPa}$ for a hybrid NiCrAl piston-cylinder cell. ${ }^{7}$ This pressure cell can generate $4.6 \mathrm{GPa}$ under a steady load, ${ }^{17}$ although the height of the sample 
space is strongly suppressed. For a clamp-type cell, where the loading force is locked by the nut to maintain pressure, the pressure usually decreases $10 \%$ after releasing the load. This NiCrAl pressure cell is powerful for the pressure-induced phenomena appearing above $3 \mathrm{GPa}$. In fact, it has been successfully applied to the following phenomena via NMR/NQR measurements: (1) pressure-induced superconductivity appearing above $3 \mathrm{GPa}$ in the telephone-number compound, $\mathrm{Sr}_{2} \mathrm{Ca}_{12} \mathrm{Cu}_{24} \mathrm{O}_{41},{ }^{18}$ and (2) the antiferromagnetic quantum critical point appearing at $4 \mathrm{GPa}$ in the heavily hydrogen-doped Fe-based compound, $\mathrm{LaFeAsO}_{1-x} \mathrm{H}_{x}(x>0.5)$. ${ }^{19}$

At high pressures between $4 \mathrm{GPa}$ and $15 \mathrm{GPa}$ and larger, both opposed- and cubic-anvil cells are available. However, their sample spaces are considerably smaller than that of a piston-cylinder cell. A typical size is $\phi 2$ in diameter and $1.0 \mathrm{~mm}-1.5 \mathrm{~mm}$ in length at the initial setup. The opposed-anvil cell is very compact in size and can be inserted into a superconducting magnet. In contrast to the opposed-anvil cell, the cubic-anvil cell is quite large and is not available for measurements under a magnetic field. In fact, the cubicanvil cell has been used mainly for resistivity or alternating-current (AC) susceptibility $(\chi)$ and recently for neutron-scattering measurements at zero field. ${ }^{20}$ As a disadvantage of the opposed-anvil cells, they require technical skills during the initial setup. Furthermore, the height of the cylindrical sample space becomes significantly short at high pressures for the opposed-anvil cell.

To downsize the cubic-anvil cell, several trials have been accomplished thus far. (1) A mini cubic-anvil cell with $\phi 94$ guide blocks was used for ${ }^{63} \mathrm{Cu}-\mathrm{NQR}$ measurements on $\mathrm{Cu}_{2} \mathrm{O} .{ }^{11}$ (2) A "palm" cubic-anvil cell (PCAC) having $\phi 80$ guide blocks was recently developed as a clamp-type cell and applied to resistivity and $\mathrm{AC}-\chi$ measurements. In this pressure cell, pressure rises to $17 \mathrm{GPa}$ by using an "integrated-fin" gasket for 2.5 -mm square-top anvils. ${ }^{21}$

In this work, we fabricated the smallest PCAC to date using $\phi 60$ $\mathrm{NiCrAl}$ guide blocks and investigated pressure homogeneity from the ${ }^{63} \mathrm{Cu}-\mathrm{NQR}$ linewidth of $\mathrm{Cu}_{2} \mathrm{O}$.

\section{PCAC}

\section{A. Design of a small PCAC}

Figure 1(a) shows a sectional view of the clamp-type PCAC, and Fig. 1(b) is a bird's-eye view of three WC anvils mounted on the $\phi 60$ $\mathrm{NiCrAl}$ guide block. Figure 1 (c) shows a photo of the NiCrAl guide blocks and $\mathrm{CuBe}$ sleeves. The size of the outer $\mathrm{CuBe}$ body is $\phi 80$ $\times 143 \mathrm{~mm}^{2}$, and the total length of the PCAC is $159 \mathrm{~mm}$. The upper and lower WC anvils having 4.0-mm square tops are directly fitted in the guide blocks. The other four WC anvils are fitted in the $\mathrm{NiCrAl}$ sliding blocks covered with Teflon sheets. The six anvils move simultaneously toward the center cube gasket when the uniaxial load is imposed on the NiCrAl guide blocks. As shown in Fig. 2(a), we prepare the upper and lower pyrophyllite gaskets, a Teflon capsule, and a Teflon cap. The Teflon capsule placed on the lower pyrophyllite gasket is shown in Fig. 2(b). A single coil wound around $\mathrm{Cu}_{2} \mathrm{O}$ power samples is inserted into the Teflon capsule for NQR measurements. In this work, we use a mixture of fluorinert FC-70 and FC-77 (1:1 by volume) as the pressure-transmitting medium. The assembly of the coil and samples are filled with the mixture of fluorinert in the Teflon capsule [see Fig. 2(c)]. The Teflon cap is placed on the

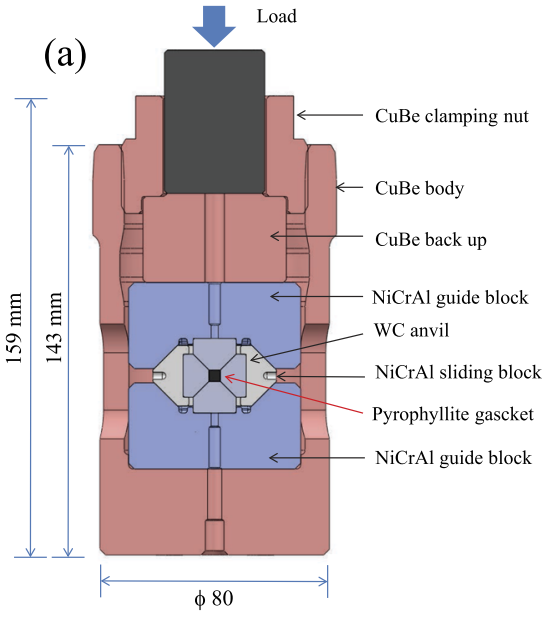

(b)

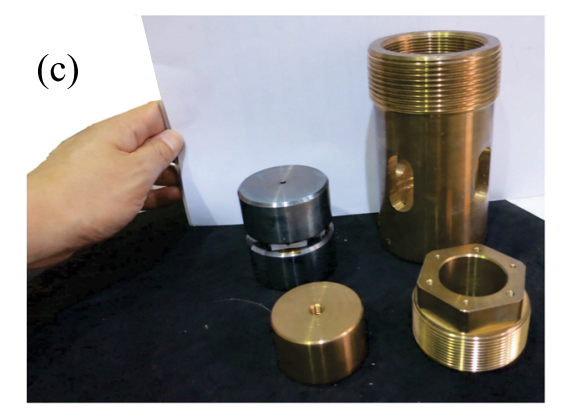

FIG. 1. (a) Sectional view of a "palm" cubic-anvil cell (PCAC) with $\phi 60$ NiCrAl guide blocks. (b) A bird's-eye view of three WC anvils and a pyrophyllite cube mounted on the lower NiCrAl guide block. (c) Photos of the NiCrAl guide blocks and $\mathrm{CuBe}$ sleeves.

capsule to avoid leakage of the mixture. Placing another pyrophyllite gasket on them, the two combined gaskets form a $6.0-\mathrm{mm}$ cube. The leads of the $\mathrm{Cu}$ coil are taken out through the gap between the anvils. The cube is sandwiched with six WC anvils, as shown in Fig. 1(a). Figure 1(b) shows the pyrophyllite cube and the three WC anvils mounted on the lower NiCrAl guide block. Notably, the setup is very easy and is free of epoxy gluing.

\section{B. Size dependence of Teflon capsules}

The resistivity of $\mathrm{Bi}$ was measured up to 85 ton at room temperature, as shown in the inset of Fig. 3 . The results were almost the same as those of the $\phi 80$ guide blocks. A $\phi 2.0$ capsule having a $\phi 1.5$ sample space is sufficient for carrying out resistivity measurements, as shown in the inset of Fig. 2(c). However, one of our purposes is to enlarge the sample space for other measurements, such as NQR. To investigate the influence of the size, we measured the resistivity for $\phi 2.0, \phi 2.5$, and $\phi 3.0$ capsules having $\phi 1.5, \phi 2.0$, and $\phi 2.5$ sample spaces, respectively. The results were nearly the same as those shown in the main panel of Fig. 3. Therefore, a large-size Teflon capsule is convenient for other experimental techniques. Hereafter, we use a $\phi 3.0$ Teflon capsule for the NQR measurements. 
(a)

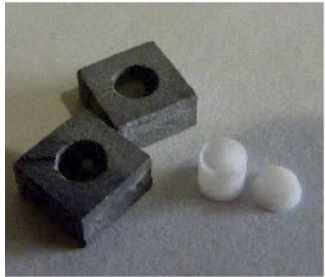

(b)

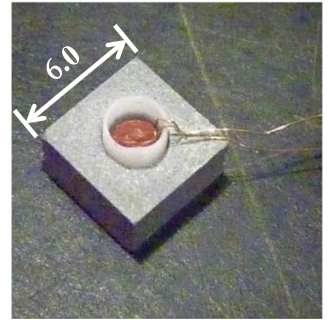

(c)

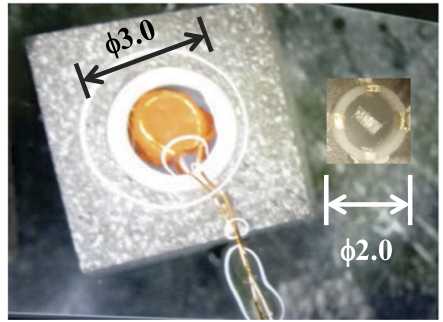

FIG. 2. (a) Photos of the upper and lower pyrophyllite gaskets, a Teflon capsule, and a Teflon cap. (b) A bird's-eye view of a $\phi 3.0$ Teflon capsule mounted on a 6.0-mm square pyrophyllite gasket. An NQR coil wrapped around powder samples of $\mathrm{Cu}_{2} \mathrm{O}$ is inserted into the capsule. (c) Overview of the $\phi 3.0$ Teflon capsule on the gasket. The inner diameter of the capsule is $\phi 2.5$. The samples and coil were immersed into the pressure-transmitting medium, i.e., a mixture of fluorinert FC-70 and FC-77 (1:1 by volume). The inset shows the $\phi 2.0$ Teflon capsule used for the resistivity measurements.

\section{Pressure calibration}

\section{Ruby fluorescence}

For piston-cylinder, diamond-anvil, and a type of opposedanvil $^{9}$ cells, the ruby-fluorescence measurement is a very powerful pressure-calibration method because it does not require any sweeps of physical parameters and in situ measurement is possible. The ruby fixed atop the optical fiber is inserted into the sample space for a piston-cylinder cell. In the ruby-fluorescence measurements below $20 \mathrm{GPa}$, the shift of the R1 line $(\Delta \lambda)$ is proportional to pressure, ${ }^{22,23}$

$$
P(G P a)=2.74 \Delta \lambda(n m) \text {. }
$$

We used Eq. (1) to determine the values of pressure for each NQR spectrum in Fig. 7 as described later.

\section{2. ${ }^{63} \mathrm{CU} N Q R$}

Since the standard ruby calibration is not possible for cubicanvil cells, ${ }^{63} \mathrm{Cu}-\mathrm{NQR}$ measurements on $\mathrm{Cu}_{2} \mathrm{O}$ serve as a pressurecalibration method, provided an NMR spectrometer is available. ${ }^{24}$

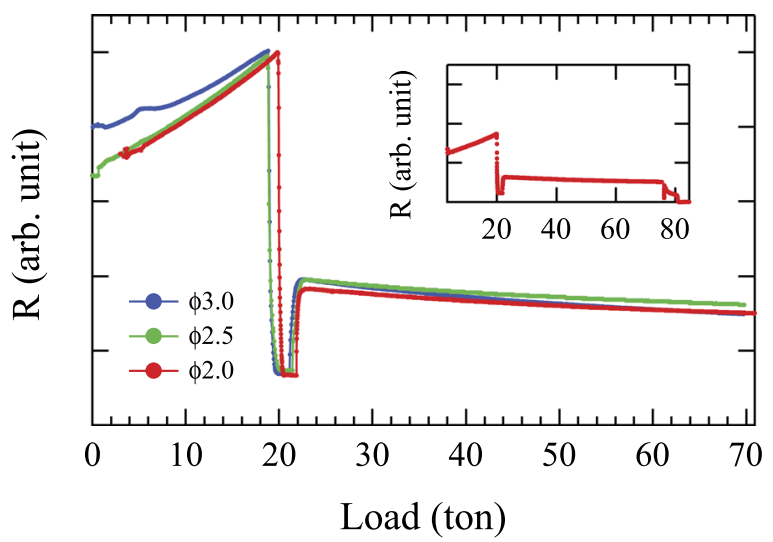

FIG. 3. Resistivity of Bi measured using the PCAC with the $\phi 60$ guide blocks. Teflon capsules having $2.0-\mathrm{mm}, 2.5-\mathrm{mm}$, and $3.0-\mathrm{mm}$ outer diameters are used for the measurements. The inset shows the resistivity for the $3.0-\mathrm{mm}$ diameter capsule up to 85 ton.
This method is convenient because it can be operated at room temperature without a magnet, unlike NMR measurements. The NQR frequency $(v)$ corresponding to the nuclear transition between $\left|\frac{3}{2}, \pm \frac{3}{2}>\Leftrightarrow\right| \frac{3}{2}, \pm \frac{1}{2}>$ shows a linear relation with pressure [see Eq. (2)]. For $I=\frac{3}{2}, v$ is expressed as $v=e^{2} q Q / 2 \hbar$, where $Q$ is the nuclear quadrupole moment and $q$ is the electric field gradient (EFG) at a nuclear site. The EFG is determined by the surrounding ions, and it is proportional to the inverse of the volume. ${ }^{25}$ The linear relation implies that the compressibility of $\mathrm{Cu}_{2} \mathrm{O}$ is pressure independent.

For a $\mathrm{NiCrAl}$ piston-cylinder cell, $v$ and $\Delta \lambda$ are in a linear relation up to 3.0 GPa from in situ measurements, and therefore, $v$ shows a linear relation with pressure as

$$
v(M H z)=26.0+0.33 P(G P a) .
$$

The $P$ dependence of $v$ is consistent with that obtained for the opposed-anvil cell up to $8.8 \mathrm{GPa}$. ${ }^{9}$ Hereafter, we use Eq. (2) to determine the pressure for the present PCAC.

\section{RESULTS OF ${ }^{63} \mathrm{Cu}$-NQR MEASUREMENTS OF $\mathrm{Cu}_{2} \mathrm{O}$}

Figure 4 shows the ${ }^{63} \mathrm{Cu}-\mathrm{NQR}$ spectra of $\mathrm{Cu}_{2} \mathrm{O}$ measured at room temperature with a conventional pulsed-NMR spectrometer and the $\phi 60$ PCAC. The spectra were obtained using the fast Fourier transform of the spin-echo signal following a sequence of two radiofrequency pulses. We applied pressure to the PCAC mounted on a 100 -ton conventional oil press. The coil having a $1.94-\mathrm{mm}$ outer diameter and a $1.5-\mathrm{mm}$ length was made using a $\phi 0.07 \mathrm{Cu}$ wire and was placed into the $\phi 3.0$ Teflon capsule. We measured the NQR spectra up to $6.74 \mathrm{GPa}$. The values of pressure for each spectrum were calculated from Eq. (2). The values would increase at low temperatures since we used the clamp-type pressure cell. For the similar PCAC with larger guide blocks, ${ }^{21}$ the pressure difference between room temperature and low temperature is within $2 \mathrm{GPa}$, and thus, a similar pressure difference is expected for the present PCAC.

Figures 5 and 6 show the load dependence of $v$ and the half linewidth at half maximum, respectively. The linewidth is proportional to the pressure inhomogeneity, as seen from Eq. (2). 


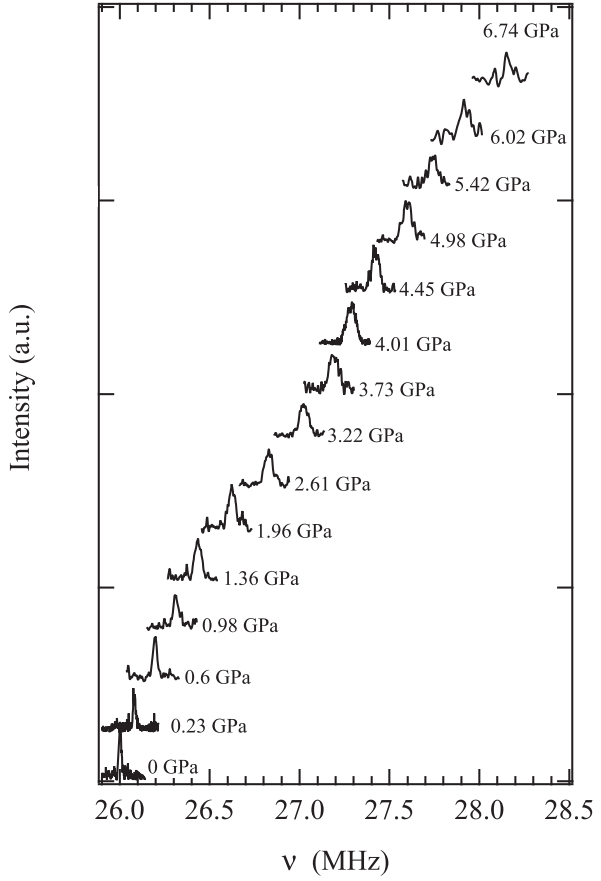

FIG. 4. ${ }^{63} \mathrm{Cu}-\mathrm{NQR}$ spectra of $\mathrm{Cu}_{2} \mathrm{O}$ measured with the $\mathrm{PCAC}$ at room temperature. The values of pressure for each spectrum were calculated using Eq. (2).

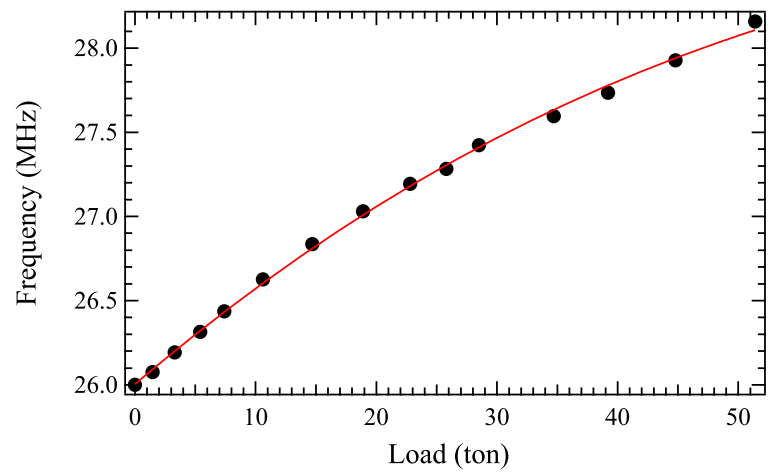

FIG. 5. Load dependence of the NQR frequency $(v)$ for the NQR spectra shown in Fig. 4. The curve is a guide to the eye.

The quality of the homogeneity is compared with that of the $\mathrm{NiCrAl}$ piston-cylinder cell in Sec. IV.

\section{DISCUSSION}

To investigate the quality of the homogeneity, we compared the present results with those for the NiCrAl piston-cylinder cell. We measured the spectra using a mixture of FC-70 and FC-77 (1:1 by volume) for comparison with the PCAC. Figure 7 shows the results of the ${ }^{63} \mathrm{Cu}-\mathrm{NQR}$ spectra of $\mathrm{Cu}_{2} \mathrm{O}$. The values of pressure for each spectrum were determined from in situ ruby-fluorescence

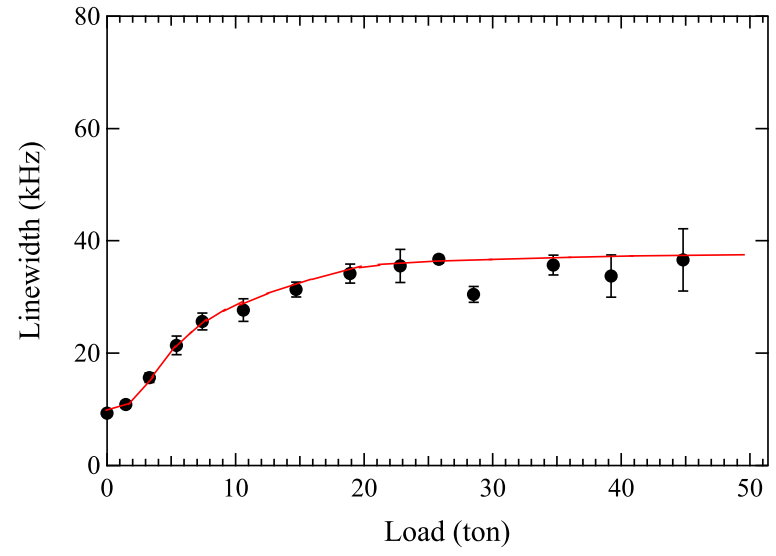

FIG. 6. Load dependence of the half width at half maximum for the NQR spectra shown in Fig. 4. The curve is a guide to the eye.

measurements [see Eq. (1)]. The signal-to-noise ratio of the spectra was better than that of the PCAC, owing to its large volume. The volume was four to five times larger than that of the PCAC. The line below $2 \mathrm{GPa}$ had a single peak. However, it showed a doublepeak structure above $2 \mathrm{GPa}$. We performed the measurements twice,

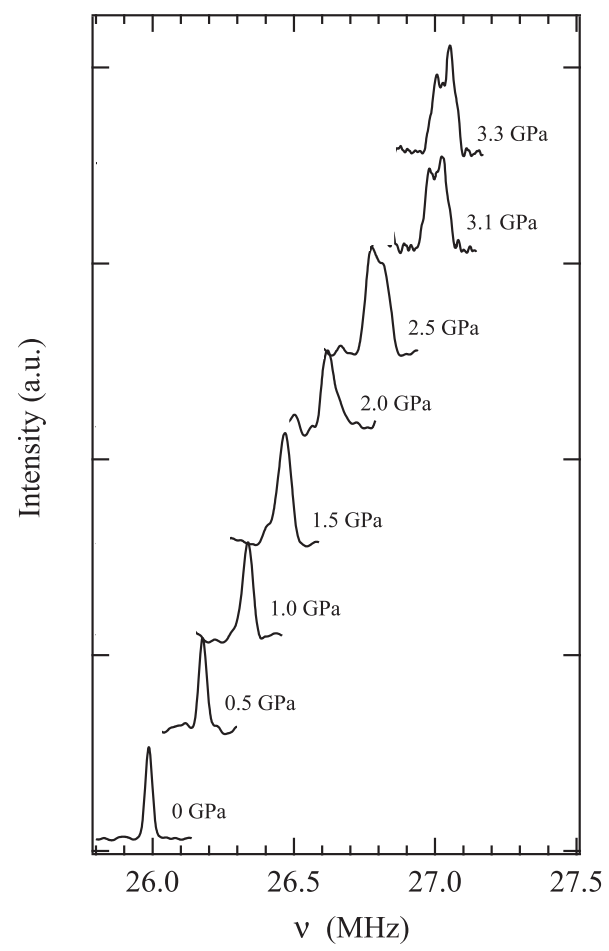

FIG. 7. ${ }^{63} \mathrm{Cu}-\mathrm{NQR}$ spectra of $\mathrm{Cu}_{2} \mathrm{O}$ measured at room temperature with a $\mathrm{NiCrAl}$ piston-cylinder cell. The values of pressure for each spectrum were determined from Eq. (1). 


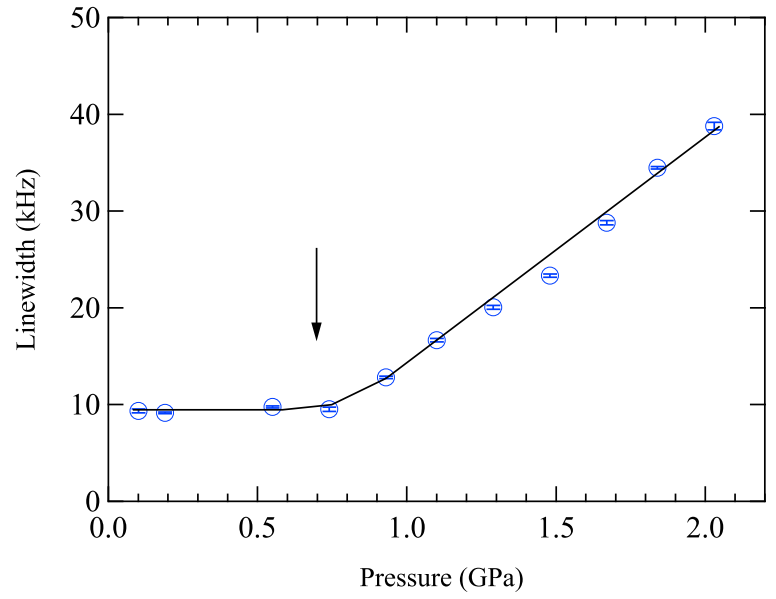

FIG. 8. Pressure dependence of the half width at half maximum for the NQR spectra shown in Fig. 7. The curve is a guide to the eye. Pressure was calibrated using the in situ ruby-fluorescence measurements. The arrow indicates the solidification of the pressure-transmitting medium (a mixture of fluorinert FC-70 and FC-7).

and the structure was reproducible. We fitted the data using a single Gaussian function for the spectra below $2 \mathrm{GPa}$. At pressures above $2 \mathrm{GPa}$, it is not certain whether the Gaussian fitting with two components makes sense because we do not know the grounds why the spectra at high pressures should have double peaks. Therefore, we estimated the linewidth at pressures below $2 \mathrm{GPa}$. The half width at half maximum is also shown in Fig. 8. As shown by the arrow, the linewidth was almost the same below $0.8 \mathrm{GPa}$, implying that the pressure-transmitting medium maintained a liquid state and high hydrostaticity. However, the mixture began to solidify above $0.8 \mathrm{GPa}{ }^{26}$ It became a sherbet-like mixture of solid and liquid states,

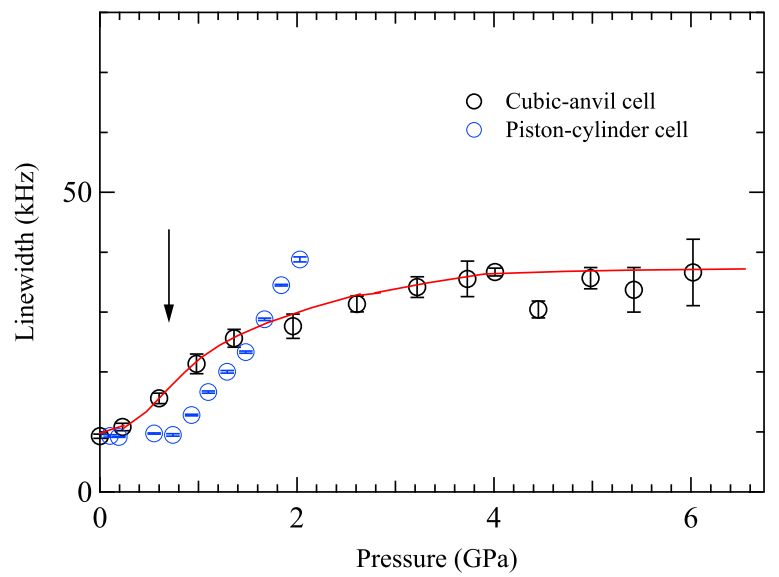

FIG. 9. Half width at half maximum of ${ }^{63} \mathrm{Cu}-\mathrm{NQR}$ spectra of $\mathrm{Cu}_{2} \mathrm{O}$ measured using the PCAC (black open circles) and NiCrAl piston-cylinder cell (blue open circles). The curve is a guide to the eye. The arrow indicates the pressure where the mixture of fluorinert FC-70 and FC-77 begins to solidify. and its inhomogeneity is increased with increasing pressure. Unlike the piston-cylinder cell, the anomaly at $0.8 \mathrm{GPa}$ was absent or negligibly small for the PCAC (Fig. 9), suggesting high hydrostaticity even at high pressures. The effect of the mixed state depended on how the load was applied to the pressure cells. Pressure application from multiple directions would suppress the inhomogeneity much more effectively than uniaxial pressure application. At the present stage, it is not certain whether the PCAC is free from the effect of solidification. In the present measurements, we demonstrated that high hydrostaticity is realized even at high pressures by using the PCAC. To investigate whether further high hydrostaticity is possible or not, NQR measurements using a highly hydrostatic medium (e.g., Daphne $7474^{27}$ ) would be needed.

\section{CONCLUSION}

We fabricated a PCAC having a diameter of $60 \mathrm{~mm}$, the smallest cubic-anvil cell to date, which allowed us to measure physical properties under a magnetic field utilizing a superconducting magnet with a large-bore sample space greater than $100 \mathrm{~mm}$. In this pressure cell, a large sample space having $\phi 2.5 \times 1.5 \mathrm{~mm}^{2}$ was secured. The size was fairly large for a pressure cell that can reach a high pressure above $4 \mathrm{GPa}$. The pressure homogeneity was better than that of the piston-cylinder cell. This pressure cell opens a new avenue for studying the pressure-induced quantum phenomena with the microscopic probes such as NMR or NQR at elevated pressure ranges.

\section{ACKNOWLEDGMENTS}

The authors thank Y. Kohori for discussions about NQR techniques under pressure. This work was supported by Grants-in-Aid for Scientific Research (KAKENHI Grant No. JP18H01181) and a grant from the Mitsubishi Foundation. This work was partly supported by Grants-in-Aid for Scientific Research (KAKENHI Grant No. JP19H00648). J.C. acknowledges the support from the National Key R\&D Program of China (Grant No. 2018YFA0305700).

\section{DATA AVAILABILITY}

The data that support the findings of this study are available from the corresponding author upon reasonable request.

\section{REFERENCES}

${ }^{1}$ H. Takahashi, K. Igawa, K. Arii, Y. Kamihara, M. Hirano, and H. Hosono, Nature 453, 376 (2008)

${ }^{2}$ H. Takahashi, A. Sugimoto, Y. Nambu, T. Yamauchi, Y. Hirata, T. Kawakami, M. Avdeev, K. Matsubayashi, F. Du, C. Kawashima, H. Soeda, S. Nakano, Y. Uwatoko, Y. Ueda, T. J. Sato, and K. Ohgushi, Nat. Mater. 14, 1008 (2015).

${ }^{3}$ J. Sun, K. Matsuura, G. Ye, Y. Mizukami, M. Shimozawa, K. Matsubayashi, M. Yamashita, T. Watashige, S. Kasahara, Y. Matsuda, J. Yan, B. Sales, Y. Uwatoko, and J. G. C. T. Shibauchi, Nat. Commun. 7, 12146 (2016).

${ }^{4}$ K. Matsuura, Y. Mizukami, Y. Arai, Y. Sugimura, N. Maejima, A. Machida, T. Watanuki, T. Fukuda, T. Yajima, Z. Hiroi, K. Y. Yip, Y. C. Chan, Q. Niu, S. Hosoi, K. Ishida, K. Mukasa, S. Kasahara, J. G. Cheng, S. K. Goh, Y. Matsuda, Y. Uwatoko, and T. Shibauchi, Nat. Commun. 8, 1143 (2017).

${ }^{5}$ J. P. Sun, P. Shahi, H. X. Zhou, Y. L. Huang, K. Y. Chen, B. S. Wang, S. L. Ni, N. N. Li, K. Zhang, W. G. Yang, Y. Uwatoko, G. Xing, J. Sun, D. J. Singh, K. Jin, 
F. Zhou, G. M. Zhang, X. L. Dong, Z. X. Zhao, and J. G. Cheng, Nat. Commun. 9, 380 (2018).

${ }^{6}$ M. I. Eremets, High Pressure Experimental Methods (Oxford, New York, 1996).

${ }^{7}$ N. Fujiwara, T. Matsumoto, K. Nakazawab, A. Hisada, and Y. Uwatoko, Rev. Sci. Instrum. 78, 073905 (2007).

${ }^{8}$ H. Fukazawa, N. Yamatoji, Y. Kohori, C. Terakura, N. Takeshita, Y. Tokura, and H. Takagi, Rev. Sci. Instrum. 78, 015106 (2007).

${ }^{9}$ K. Kitagawa, H. Gotou, T. Yagi, A. Yamada, T. Matsumoto, Y. Uwatoko, and M. Takigawa, J. Phys. Soc. Jpn. 79, 024001 (2010).

${ }^{10}$ T. Meier, N. Wang, D. Mager, J. G. Korvink, S. Petitgirard, and L. Dubrovinsky, Sci. Adv. 3, eaao5242 (2017).

${ }^{11}$ K. Hirayama, T. Yamazaki, H. Fukazawa, Y. Kohori, and N. Takeshita, J. Phys. Soc. Jpn. 77, 075001 (2008).

${ }^{12}$ R. Bertani, M. Mali, J. Roos, and D. Brinkmann, Rev. Sci. Instrum. 63, 3303 (1992).

${ }^{13}$ M. G. Pravica and I. F. Silvera, Phys. Rev. Lett. 81, 4180 (1998).

${ }^{14}$ M. G. Pravica and I. F. Silvera, Rev. Sci. Instrum. 69, 479 (1998).

${ }^{15}$ T. Meier and J. Haase, Rev. Sci. Instrum. 86, 123906 (2015).

${ }^{16}$ T. Meier, F. Trybel, S. Khandarkhaeva, G. Steinle-Neumann, S. Chariton,

T. Fedotenko, S. Petitgirard, M. Hanfland, K. Glazyrin, N. Dubrovinskaia, and

L. Dubrovinsky, Phys. Rev. X 9, 031008 (2019).
${ }^{17}$ N. Fujiwara, Y. Uwatoko, and T. Matsumoto, J. Phys.: Conf. Ser. 500, 032008 (2014).

${ }^{18}$ N. Fujiwara, N. Mori, Y. Uwatoko, T. Matsumoto, T. Motoyama, and S. Uchida, Phys. Rev. Lett. 90, 137001 (2003).

${ }^{19}$ M. Takeuchi, N. Fujiwara, T. Kuwayama, S. Nakagawa, S. Iimura, S. Matsuishi, Y. Yamakawa, H. Kontani, and H. Hosono, Phys. Rev. B 99, 174517 (2019).

${ }^{20}$ S. E. Dissanayake, M. Matsuda, K. Munakata, H. Kagi, J. Gouchi, and Y. Uwatoko, J. Phys.: Condens. Matter 31, 384001 (2019).

${ }^{21}$ J.-G. Cheng, K. Matsubayashi, S. Nagasaki, A. Hisada, T. Hirayama, M. Hedo, H. Kagi, and Y. Uwatoko, Rev. Sci. Instrum. 85, 093907 (2014).

${ }^{22}$ G. J. Piermarni, S. Block, J. D. Barnett, and R. A. Forman, J. Appl. Phys. 46, 2774 (1975).

${ }^{23}$ H. K. Mao, P. M. Bell, J. W. Shaner, and D. J. Steinberg, J. Appl. Phys. 49, 3276 (1978).

${ }^{24}$ A. P. Reyes, E. T. Ahrens, R. H. Heffner, P. C. Hammel, and J. D. Thompson, Rev. Sci. Instrum. 63, 3120 (1992)

${ }^{25}$ T. Kushida, G. B. Benedek, and N. Bloembergen, Phys. Rev. 104, 1364 (1956).

${ }^{26}$ V. A. Shidorov and R. A. Sadykov, J. Phys.: Condens. Matter 17, S3005 (2005).

${ }^{27}$ K. Murata, K. Yokogawa, H. Yoshino, S. Klotz, P. Munsch, A. Irizawa, M. Nishiyama, K. Iizuka, T. Nanba, T. Okada, Y. Shiraga, and S. Aoyama, Rev. Sci. Instrum. 79, 085101 (2008). 